\title{
Dispersion polymerization of methyl methacrylate in supercritical carbon dioxide using vinyl terminated poly(dimethylsiloxane)
}

\author{
P.F. Oliveira ${ }^{\mathrm{a}, \mathrm{b}}$, R.A.F. Machado ${ }^{\mathrm{a}, *}$, D. Barth ${ }^{\mathrm{b}}$, E.D. Acosta ${ }^{\mathrm{a}}$ \\ a Universidade Federal de Santa Catarina, Departamento de Engenharia Química e Engenharia de Alimentos, Brazil \\ ${ }^{\mathrm{b}}$ Université de Lorraine, Laboratoire Réactions et Génie des Procedés (LRGP), UMR 7274, France
}

\section{A R T I C L E I N F O}

\section{Article history:}

Received 3 July 2015

Received in revised form 20 October 2015

Accepted 27 October 2015

Available online 10 November 2015

\section{Keywords:}

Dispersion polymerization

Supercritical fluids

Siloxane macromonomers

\begin{abstract}
A B S T R A C T
PMMA has applications on the medical, pharmaceutical and engineering areas. Different processes may be employed for its production. Here, MMA polymerization was performed by supercritical carbon dioxide dispersion. $\mathrm{CO}_{2}$ is inert, easily retrievable and separated from the product. The monomer is soluble in dense $\mathrm{CO}_{2}$, unlike PMMA, requiring a stabilizer to maintain the dispersion. Here, vinyl terminated PDMS was used as a dispersing agent. The reactions were conducted at $16 \mathrm{MPa} / 80^{\circ} \mathrm{C}$ for $4 \mathrm{~h}$. Product characterization was performed by SEM, to evaluate the morphology at estimate the particle size, SEC, to determine the molar mass and polydispersion index, ${ }^{1} \mathrm{H}$ NMR and FTIR spectroscopy, in order to verify the chemical structure of the product, and also gravimetric analyses, to determine the residual monomer. The results confirmed the adequacy of the process and of the stabilizer for PMMA particles production.
\end{abstract}

(c) 2015 Elsevier B.V. All rights reserved.

\section{Introduction}

Poly(methyl methacrylate), PMMA, is a widely used polymer for engineering, medical and pharmaceutical purposes. Depending on the desired final application suspension, solution, emulsion, dispersion or bulk polymerization processes can be used to obtain PMMA. Except for mass/bulk polymerization and dispersion in $\mathrm{scCO}_{2}$, all the other techniques utilize water or organic solvents in the process. Dispersion polymerization using supercritical fluids have received considerable interest of many researchers [1-7]. Mostly studied systems consist of vinyl monomers, methyl methacrylate and styrene, in $\mathrm{scCO}_{2}[1-6,8]$. For these applications, $\mathrm{scCO}_{2}$ dispersion polymerization can be used as a procedure to produce latexes, as well as spherical particles, thus representing a versatile polymerization system.

Carbon dioxide is mostly applied as a medium, since it is not expensive, inert, easily retrievable and separated from the product. The great part of monomers and polymers are not soluble in $\mathrm{CO}_{2}$. At elevated pressures, the solubility of monomers in $\mathrm{CO}_{2}$ increase, being possible to adjust this property by changes in both pressure and temperature values. As the polymer chain grows, up to a certain limit, solubility in $\mathrm{scCO}_{2}$ decreases and particle formation occurs by precipitation. To promote a good dispersion along the

\footnotetext{
* corresponding author.

E-mail address: ricardo.machado@ufsc.br (R.A.F. Machado).
}

reaction it is necessary to use a surfactant. Fluorinated and siloxane based macromonomers have been extensively applied as stabilizing agents in dispersed systems using $\mathrm{scCO}_{2}$ for the $\mathrm{CO}_{2}$-philic characteristics and the ability to stabilize polymer growing chains $[2,3,7]$. Since $\mathrm{CO}_{2}$ is inert it presents advantages in polymerization systems, due to the absence of chain transfer to the solvent [9]. Dispersion polymerization systems favor the formation of stable particles in supercritical medium and the obtention of higher molar masses and conversions [10].

The application of dispersion polymerization in supercritical $\mathrm{CO}_{2}$ to produce very fine particles presents advantages such as particle size narrow distribution and the obtention of highly pure polymers, given that by depressurizing the system, the solvent is eliminated $[3,11]$. In the supercritical fluid, amorphous polymers are expanded and plasticized, thus reducing their glass transition temperature. The effects of supercritical $\mathrm{CO}_{2}$ on these polymers also include increased chain mobility and improvement of monomer diffusion, favoring the elimination of residual monomer, additive incorporation and foam formation [11]. The polymerization by dispersion process distinguishes itself from the classic polymerization in suspension process as it consists, initially, of a homogeneous system containing monomer, organic solvent, a soluble initiator in the dispersed phase and stabilizing agent. The system becomes heterogeneous throughout the reaction, as the formed polymer is insoluble in solvent (supercritical $\mathrm{CO}_{2}$ ).

In this work, methyl methacrylate (MMA) polymerization was performed in dispersed medium, using carbon dioxide $\left(\mathrm{CO}_{2}\right)$ in 
supercritical conditions. Vinyl terminated poly(dimethylsiloxane) was used to stabilize the reaction, a commercial macromonomer. The use of a commercially available stabilizer may have important implication on the scale up of the supercritical polymerization process. The experimental conditions were based on researches conducted using methyl methacrylate dispersion polymerization in supercritical $\mathrm{CO}_{2}$ using different operating conditions and siloxane based stabilizers $[1,2,4]$.

Methyl methacrylate is soluble in $\mathrm{CO}_{2}$ under conditions above $80^{\circ} \mathrm{C}$ and $12 \mathrm{MPa}$ [2]. In view that the best yield and quality results of polymer obtained by Han et al. [2] were given at pressures between 13.8 and $20.7 \mathrm{MPa}$ and that of Gilles et al. [4] was with pressure ranges within these values, the experimental setting was defined as the intermediate pressure of $16 \mathrm{MPa}$ at $80^{\circ} \mathrm{C}$. As methyl methacrylate is soluble in the operating conditions applied in this work, the MMA $/ \mathrm{CO}_{2}$ system is homogeneous during the beginning of the polymerization reaction. As the reaction occurs, the formed polymeric chains are expected to precipitate as the system MMA/ $\mathrm{CO}_{2} /$ PMMA becomes heterogeneous. The product is obtained as microspheres of PMMA.

\section{Materials and methods}

\subsection{Polymerization reactions}

The methyl methacrylate polymerization reactions in supercritical media were performed in a jacketed stainless steel reactor (Top Industrie, France), with $100 \mathrm{~mL}$ internal volume, internal diameter and height of 40 and $80 \mathrm{~mm}$, respectively. The compression module consists of a $\mathrm{CO}_{2}$ cylinder, a controlled temperature water bath, membrane pump, heat exchanger and a mass flow meter. The reaction temperature is measured by a thermocouple inside the vessel. The dispersed system is maintained by a magnetic stirrer, which possesses a Rushton stem with scoops of $16 \mathrm{~mm}$ diameter, situated at $5 \mathrm{~mm}$ from the bottom of the reactor.
The stirring velocity was set to $900 \mathrm{rpm}$. The $\mathrm{CO}_{2}$ used has $99.99 \%$ purity. The reactants, methyl methacrylate (Fluka, $\geq 98.5 \%$ ), vinyl terminated poly(dimethyl siloxane) (Sigma-Aldrich) as the stabilizing agent and AIBN ( $\alpha, \alpha^{\prime}-$ azoisobutyronitrile; purity $98 \%$; Sigma-Aldrich) as the initiator were introduced into the high pressure vessel at $35^{\circ} \mathrm{C}$. All materials were used as received. Once the reactor is shut, it is gradually fed with $\mathrm{CO}_{2}$, simultaneously, with the increase in temperature, until the reaction conditions are reached. The $\mathrm{CO}_{2}$ supply is suspended once the system stabilizes on the required conditions. After the end of the reaction ( $4 \mathrm{~h})$, the system is cooled and depressurized to ambient conditions. The polymer, on the form of fine particles is, then, recovered with a spatula. Samples were taken from the vessel at the end of the reaction and had hydroquinone added in order to measure residual monomer by gravimetric procedure. The rest of the product was then washed with $n$-hexane, for the removal of stabilizing agent (PDMS, vinyl terminated) residue, vacuum filtered and followed by oven drying at $30^{\circ} \mathrm{C}$ with air circulation. The high pressure polymerization unit is illustrated in Fig. 1 and the experimental specifications are shown in Table 1 . All polymerization reactions were performed at least 3 times each, since instability in pressure and temperature maintenance was observed and is expected in exothermic systems. Reactions were carried out at the LRGP, Université de Lorraine, France.

\subsection{Polymer characterizations}

PMMA particles were characterized by its molar mass, residual monomer presence, morphology and particle size distribution, as well as chemical structure and functional groups. For this, the samples were subjected to Size Exclusion Chromatography (SEC), gravimetric measures, scanning electron microscopy (SEM) using SizeMeter 1.1 to analyze images and measure particles size diameter, ${ }^{1} \mathrm{H}$ NMR (Proton nuclear magnetic resonance) and FTIR (Fourier transform infrared spectroscopy), respectively. The

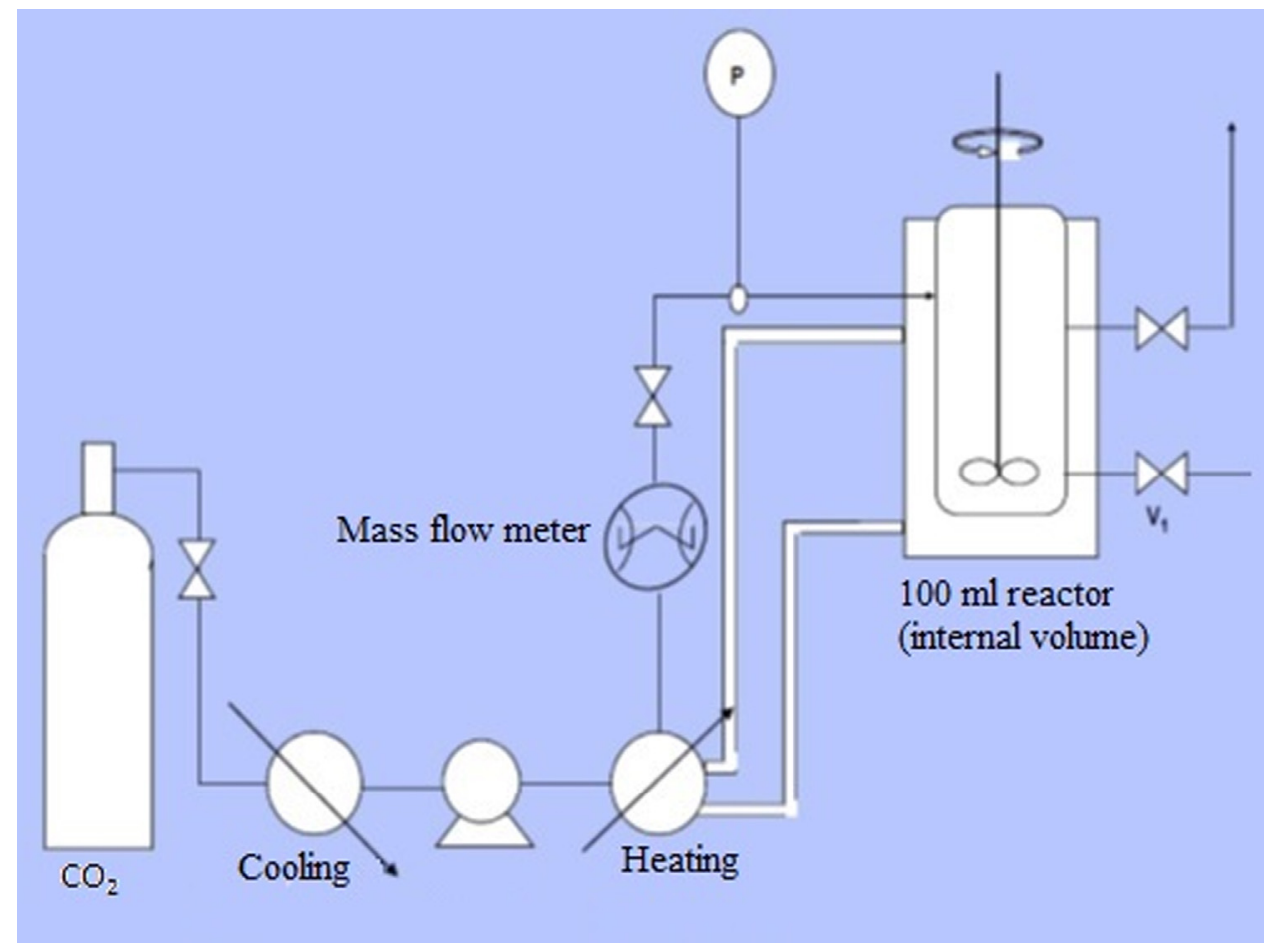

Fig. 1. High pressure polymerization unit scheme. 
Table 1

Experimental settings for MMA polymerization in supercritical $\mathrm{CO}_{2}$.

\begin{tabular}{|c|c|}
\hline \multirow[t]{4}{*}{ Reaction parameters } & $P=16 \mathrm{MPa}$ \\
\hline & $T=80^{\circ} \mathrm{C}$ \\
\hline & Stirring $=900 \mathrm{rpm}$ \\
\hline & Reaction length $=4 \mathrm{~h}$ \\
\hline \multirow[t]{7}{*}{ Formulation } & Methyl methacrylate $=28.9 \% \mathrm{w} / \mathrm{w}$, ratio to the total system mass; \\
\hline & $\rho_{\mathrm{MMA}}=940 \mathrm{~g} / \mathrm{l}\left(80^{\circ} \mathrm{C}\right)$ \\
\hline & AIBN $=0.48 \% \mathrm{w} / \mathrm{w}$, ratio to the monomer mass \\
\hline & Stabilizer: vinyl terminated PDMS, $M_{\mathrm{w}}=25,000 \mathrm{~g} / \mathrm{gmol}$ (Sigma-Aldrich) \\
\hline & Stabilizer $=5.4 \%$ and $7.02 \%$ ( $30 \%$ increase) $\mathrm{w} / \mathrm{w}$, ratio to the monomer mass \\
\hline & $m_{\mathrm{CO} 2}=38.9 \mathrm{~g}$ \\
\hline & $\rho_{\mathrm{CO} 2}=470 \mathrm{~g} / \mathrm{l}\left(16 \mathrm{MPa} / 80^{\circ} \mathrm{C}\right)$ \\
\hline
\end{tabular}

analyses were conducted at the Universite de Lorraine (LRGP and LCPM, ENSIC, France) and at the Universidade Federal de Santa Catarina (LCP/EQA, Brazil).

Molecular weight distributions were determined by Size Exclusion Chromatography, which is based on the selective retention of macromolecules. The chromatographic system used in this work is composed by an HPLC pump L-6200 A $(0.7 \mathrm{~mL} / \mathrm{min})$, an injection loop of $100 \mu \mathrm{l}$, and a series of columns Polymer Laboratories. The columns type PLgel $5 \mu \mathrm{m} 10^{5} \mathrm{~A}(300 \times 7,5 \mathrm{~mm})$ at $40{ }^{\circ} \mathrm{C}$ are preceded by a pre-column type PLgel $5 \mu \mathrm{m}$ Guard $(50 \times 7,5 \mathrm{~mm})$. At the columns output there is a photo scatterometer model MiniDawn (Wyatt Technology Corporation). The laser wavelength is set at $690 \mathrm{~nm}$ by the manufacturer and three photodiodes measure the scattered intensity at $47.6^{\circ}, 90.0^{\circ}$ and $132.4^{\circ}$. A double detection is performed using a differential refractometer type Waters 410 , installed in series. The results are collected and processed by the Astra 4.90 software. Samples were prepared in tetrahydrofuran (THF), also used as the eluent. The molecular weight distributions were determined relative to PS standards (580-3,800,000 Da).

Gravimetric analyses were performed to evaluate residual monomer in the final polymer. Samples were solubilized in chloroform p.a. (Sigma-Aldrich) and p-benzoquinone (SigmaAldrich) was added to each sample in order to inhibit polymerization reactions after the supercritical procedure. Once solubilized, samples were dried in a forced air circulation-drying oven (De Leo) at $60^{\circ} \mathrm{C}$, weighed after $12 \mathrm{~h}$ and every $4 \mathrm{~h}$ since then until constant weight was reached $(24 \mathrm{~h})$. The residual monomer was determined by the difference between initial and final weight measures.

Scanning electron microscopy (SEM) images were collected using a JEOL JSM $6490 \mathrm{LV}$ equipment with an acceleration voltage of $5 \mathrm{kV}$ and $2 \mathrm{kV}$ under high vacuum and with the secondary electron detector. Samples were prepared by depositing the particles on a double sided tape on brass studs. Next, the particles were coated with a layer of $\mathrm{Au} / \mathrm{Pd}(80-20 \%)$ by cathode sputtering (device Ion Sputter JFC 1100 from JEOL). The particle diameters of the PMMA product were averaged from measurements of individual particles of each SEM micrograph using Size Meter 1.1 software.
Proton nuclear magnetic resonance spectra were recorded on a Bruker Avance spectrometer operating at $300 \mathrm{MHz}$ in deuterated chloroform $\left(\mathrm{CDCl}_{3}\right)$. The displacements were given using tetramethylsilane (TMS) as internal standard ( $\delta=0$ ppm for TMS).

Fourier transform infrared spectroscopy analyses were performed using a Shimadzu FTIR, model IR Prestige-21, with $4 \mathrm{~cm}^{-1}$ resolution and 32 scans. Samples were evaluated in a range of $4500-400 \mathrm{~cm}^{-1}$. PMMA samples were prepared with potassium bromide $(\mathrm{KBr})$. Samples were evaluated in transmission operation mode. Stabilizer vinyl terminated PDMS was analyzed using horizontal attenuated total reflectance (HATR) (PIKE Technologies).

\section{Results and discussion}

In this item, the results obtained for the methyl methacrylate polymerization reactions by dispersion in supercritical $\mathrm{CO}_{2}$ and their product characterizations are presented and discussed. Table 2 shows the molar mass ( $\mathrm{g} / \mathrm{gmol}$ ) and the polydispersion index (PDI) obtained for the PMMA. Results are in agreement with PMMA obtained using supercritical $\mathrm{CO}_{2}$ with similar systems elsewhere $[2,4,5]$. High molecular weight polymer was obtained $\left(10^{5} \mathrm{~g} / \mathrm{gmol}\right)$, as expected for this system. The use of a stabilizing agent is required to effectively promote the free radical polymerization in supercritical $\mathrm{CO}_{2}$, since it works in the maintenance of the solubility of the growing polymer on the $\mathrm{sCCO}_{2}$, thus favoring the chain formation. According to Han et al. [1], when evaluating the stabilizer effect on dispersion polymerization of $\mathrm{MMA}$ in $\mathrm{ScCO}_{2}$, by increasing the amount of stabilizer the growing polymer chains become more stable in the solution MMA- $\mathrm{CO}_{2}$ and, thus, molecular weight increases and presents narrow distribution. When an increase of $30 \%$ on the amount of stabilizer was applied, no significant changes in molecular weight were observed, as it can be seen from runs 7 and 8 in Table 2 . The reaction is exothermic and at high pressure systems this effect is more pronounced. Wang et al. [6] demonstrated the effect of the exothermic reaction and pressure perturbation until equilibrium for MMA polymerization reaction in supercritical $\mathrm{CO}_{2}$. Eventual fluctuation on pressure in the first two hours of reaction might promote changes in the

Table 2

MMA polymerization reactions by dispersion in supercritical $\mathrm{CO}_{2}$. Operational parameters, molar mass and polydispersity index.

\begin{tabular}{|c|c|c|c|c|c|}
\hline Run No. & \% Stabilizer & $M_{\mathrm{n}} \times 10^{5}(\mathrm{~g} / \mathrm{gmol})$ & $M_{\mathrm{w}} \times 10^{5}(\mathrm{~g} / \mathrm{gmol})$ & PDI $\left(M_{\mathrm{w}} / M_{\mathrm{n}}\right)$ & Aspect of the product \\
\hline 1 & 5.4 & 1.465 & 5.279 & $3.603 \pm 0.047$ & Powder \\
\hline 2 & 5.4 & 2.468 & 4.866 & $1.972 \pm 0.022$ & Powder \\
\hline 3 & 5.4 & 1.562 & 5.317 & $3.405 \pm 0.025$ & Powder \\
\hline 4 & 5.4 & 1.222 & 3.900 & $3.192 \pm 0.295$ & Powder \\
\hline 5 & 5.4 & 1.154 & 2.368 & $2.052 \pm 0.022$ & Powder \\
\hline 6 & 5.4 & 2.409 & 4.843 & $2.011 \pm 0.026$ & Powder \\
\hline 7 & 7.02 & 1.135 & 2.314 & $2.038 \pm 0.043$ & Powder \\
\hline 8 & 7.02 & 1.302 & 2.623 & $2.015 \pm 0.054$ & Powder \\
\hline
\end{tabular}


Table 3

Results for residual MMA and average particle size.

\begin{tabular}{lll}
\hline Run No. & Residual monomer $(\%)$ & Mean particle diameter $(\mu \mathrm{m})$ \\
\hline 1 & $5.2 \pm 0.2$ & $60.57 \pm 11.70$ \\
2 & $5.2 \pm 0.5$ & $29.67 \pm 8.50$ \\
4 & $5.3 \pm 0.4$ & $30.16 \pm 12.57$ \\
5 & $5.5 \pm 0.1$ & $19.86 \pm 8.29$ \\
7 & $5.3 \pm 0.2$ & $29.63 \pm 10.53$ \\
8 & $5.5 \pm 0.3$ & $34.90 \pm 11.83$ \\
\hline
\end{tabular}

solubility of the MMA/CO $/$ /growing PMMA system and, thus, interfere on polymer chain growing, which can be associated to the differences in molecular weight and its distribution values obtained in the present work.

Rosell et al. [5] performed MMA polymerization by dispersion in supercritical $\mathrm{CO}_{2}$ using a fluorinated stabilizer, obtaining a polymer with molar mass of the order of $10^{5} \mathrm{~g} / \mathrm{gmol}$. Likewise, Han et al. [2] obtained PMMA with molar mass on the $10^{4} \mathrm{~g} / \mathrm{gmol}$ order of magnitude, using glycidyl methacrylate-linked poly(dimethylsiloxane) as the stabilizing agent. Similarly, Giles et al. [4] obtained PMMA with $M_{\mathrm{n}}$ from $10^{4}$ to $10^{5} \mathrm{~g} / \mathrm{gmol}$ order of magnitude using PDMS monomethacrylate of different $M_{\mathrm{n}}$ as a stabilizing agent of dispersion polymerization with supercritical $\mathrm{CO}_{2}$, using $1 \%$ of $\mathrm{AIBN}$.

In Table 3 are presented the results for the residual monomer and the average particle size of the final product. The maximum conversion obtained was, approximately, 95\%, in mass. Rosell et al. [5] also found elevated values of PMMA conversion (98\% maximum). The PMMA particle diameter was determined by analyzing SEM micrographs with Size Meter 1.1. The results show non-negligible variations among them. This might be caused by pressure and, consequently, temperature perturbations throughout the reactions, due to the exothermic system, the gel effect that occurs in free radical polymerization systems, as well as equipment limitations in the accurate control of both pressure and temperature parameters, as all these factors may influence the final product.
An increase of $30 \%$ of stabilizing agent was applied to runs 7 and 8 , in order to obtain smaller particles and a narrow distribution. However, this raise showed no significant changes in values, evidencing the need for higher stabilizer concentration and/or higher stirring velocity to maintain the dispersion.

In Fig. 2 the PMMA obtained in this process are shown, exhibiting the spherical shape of the particles, consistent with previous works found in the literature $[1,2,5,9,10]$. According to Wang and Cheung [13], the stabilizer concentration controls the morphology of the polymer. The polymerization process led to PMMA particles ranging from $11.57 \mu \mathrm{m}$ to $72.27 \mu \mathrm{m}$ when using $5.4 \% \mathrm{w} / \mathrm{w}$ of stabilizing agent, and from $19.1 \mu \mathrm{m}$ to $46.73 \mu \mathrm{m}$ when using $7.02 \% \mathrm{w} / \mathrm{w}$ of stabilizing agent. However, for the tests and measurements presented one cannot affirm that higher concentrations of stabilizing agent lead to smaller particle size diameters, as it is usually observed in polymerization systems.

Vinyl PDMS $\left(M_{\mathrm{w}}=25,000 \mathrm{~g} / \mathrm{gmol}\right)$ stands as a compatible stabilizer to be used for the system $\mathrm{MMA} / \mathrm{CO}_{2}$ in polymerization reactions. In the concentrations applied and for the experimental conditions adopted in this work, it favored the obtention of polydispersed PMMA particles. Other factors may also have contributed to these results, such as stirring, pressure and temperature fluctuations during reactions, thus leading to lack of stability and changes in the solubility of monomer in $\mathrm{scCO}_{2}$.

${ }^{1} \mathrm{H}$ NMR analyses of the polymer samples were performed aiming to verify the incidence of side reactions, that is, the reactivity of the stabilizing agent in the proposed system, as well as, the efficiency of the polymerization process in dense $\mathrm{CO} 2$ to obtain PMMA, as shown in Fig. 3 for one of the samples. In view that the operational parameters were not changed, it is shown only the analysis for one of the reactions. All other ${ }^{1} \mathrm{H}$ NMR spectra presented equal results. Samples were prepared in $\mathrm{CDCl} 3$, as previously described in Section 2 (Materials and methods). PDMS macromonomers are expected to behave as reactive surfactants in the steric stabilization of the CO2/MMA/PMMA system. Therefore, side reactions between stabilizer and the growing polymer,
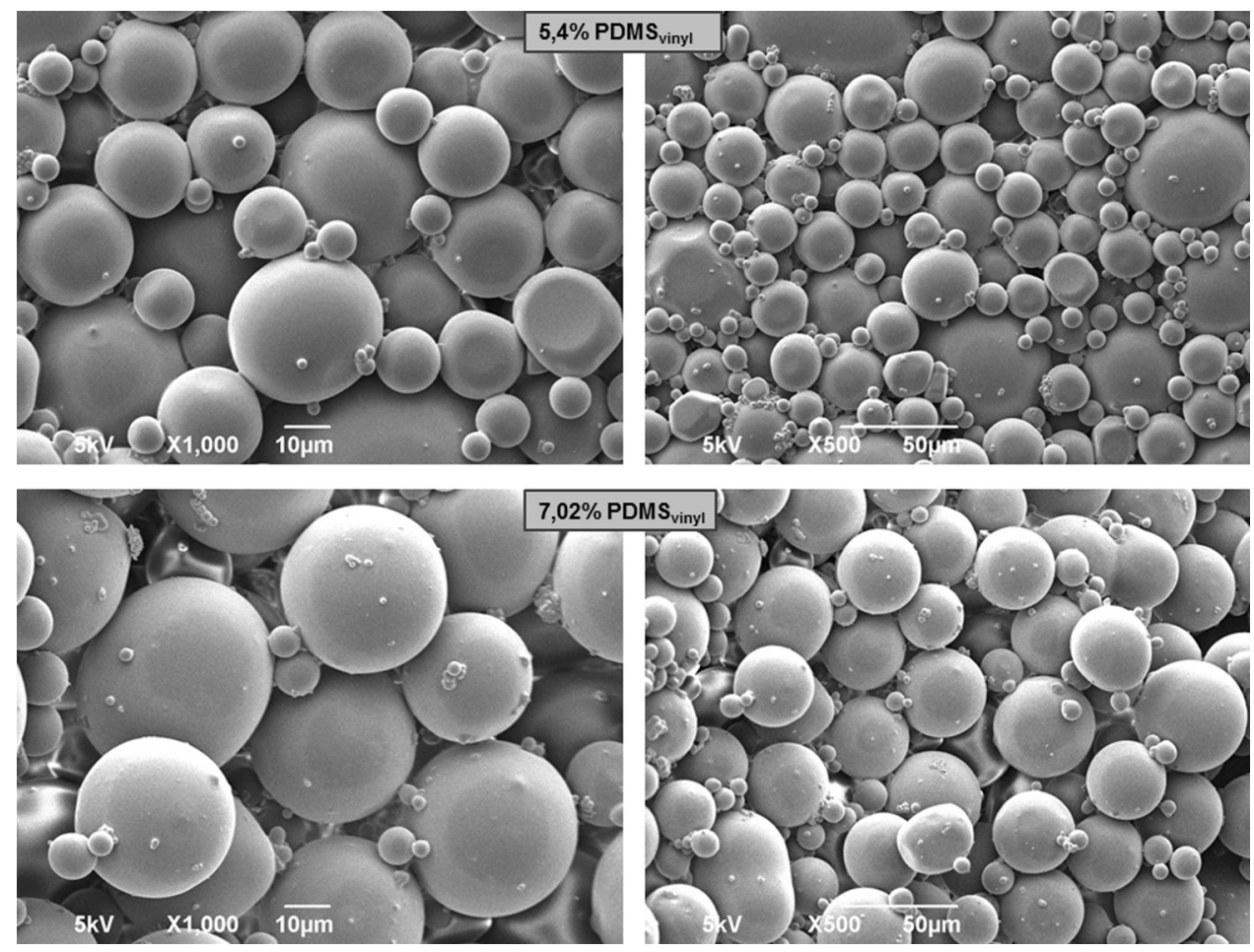

Fig. 2. PMMA SEM micrographs (magnifications of $500 \times$ and $1000 \times$ ). 


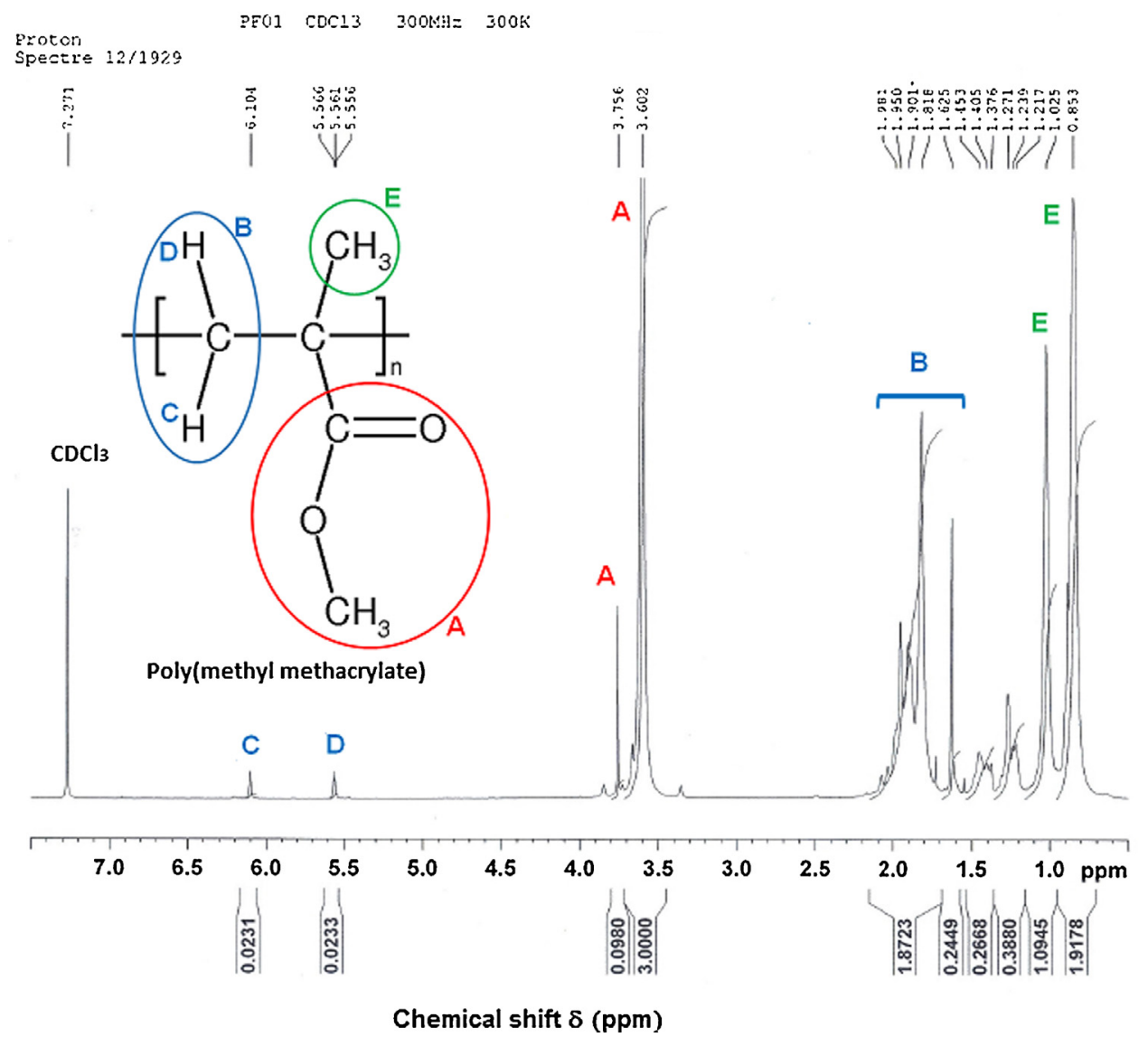

Fig. 3. ${ }^{1} \mathrm{H}$ NMR analysis for the obtained PMMA. The solvent $\left(\mathrm{CDCl}_{3}\right)$ peak at $\delta=7.271 \mathrm{ppm}$.

although not desired, may occur. The results for the analyzed samples demonstrate that PMMA was the major molecule formed in the process. Minor peaks are possibly due to residual monomer (methyl methacrylate). Hydrogens related to a supposed reaction between vinyl PDMS and MMA were not observed.
Although ${ }^{1} \mathrm{H}$ NMR, as it was performed, is not a conclusive measure to ensure the occurrence or absence of side reactions, or the formation of a copolymer between MMA and vinyl PDMS, the results here presented rise the hypothesis that no reaction occurred between MMA and PDMS, other than the stabilizing

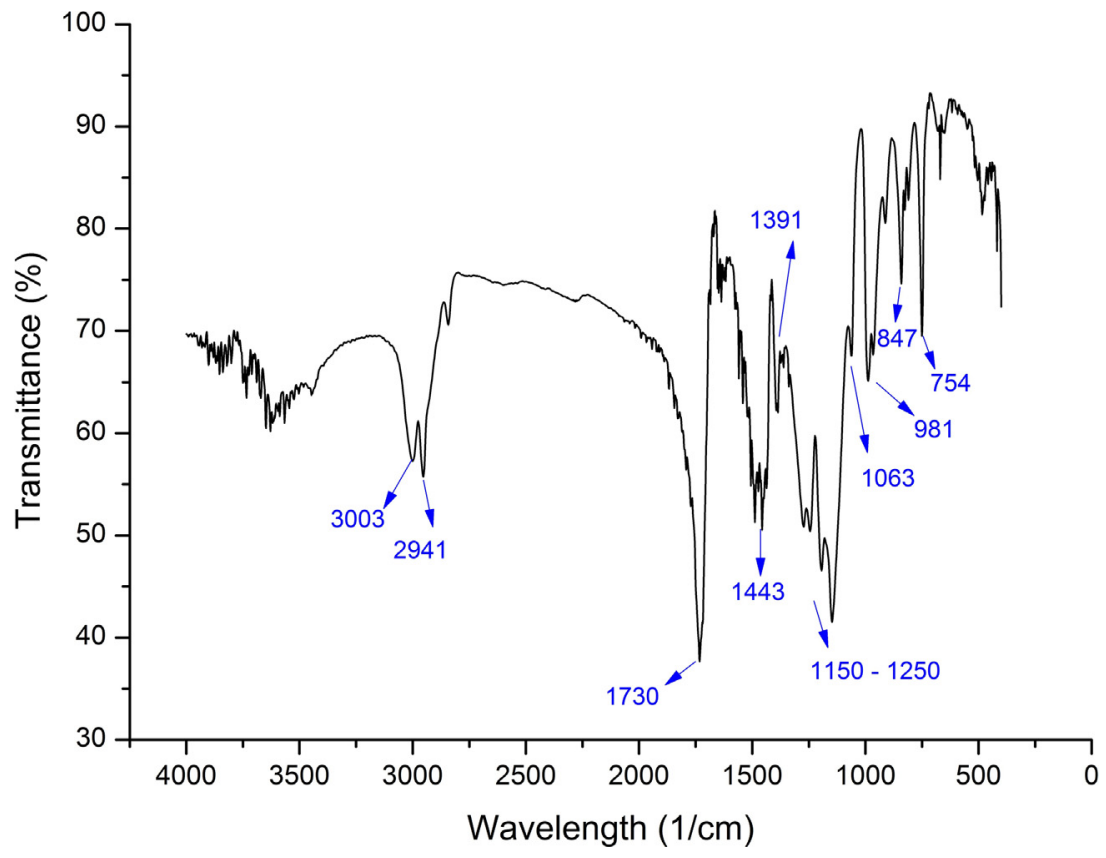

Fig. 4. FTIR spectrum of PMMA. 


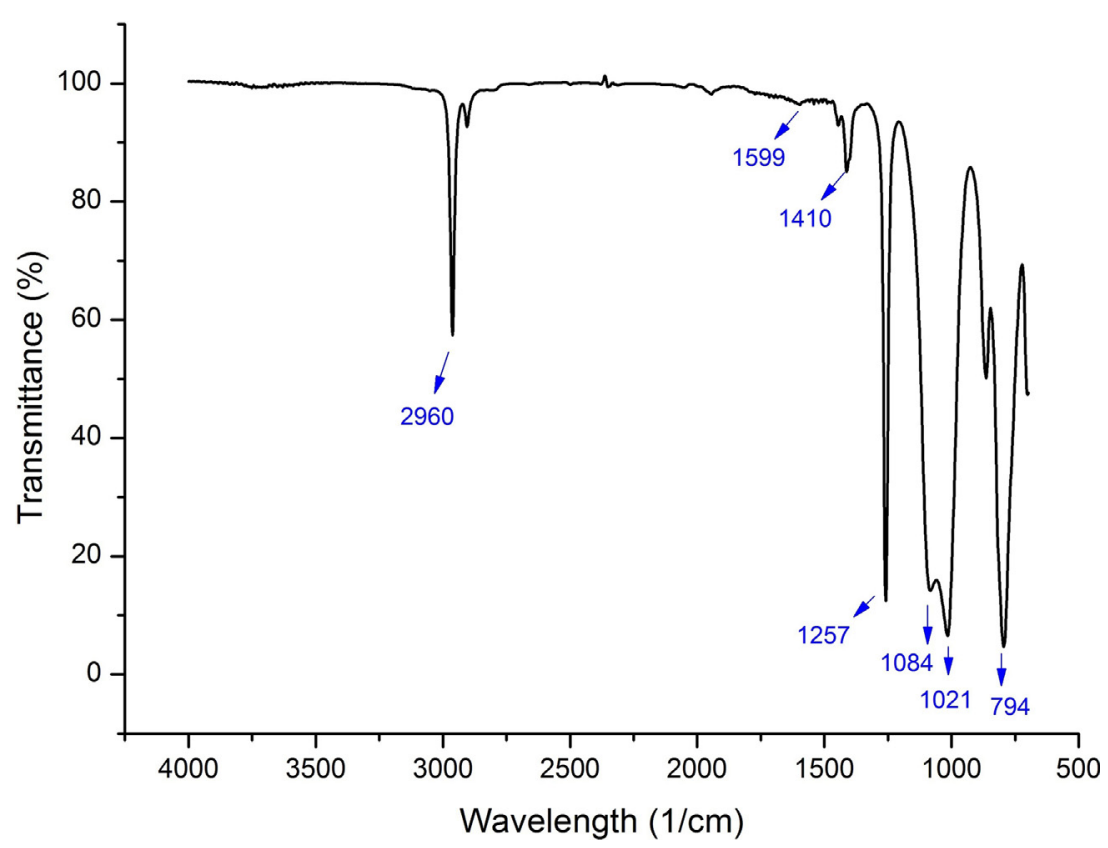

Fig. 5. FTIR spectrum of macromonomer vinyl PDMS.

effect. This fact stands as a positive result for the use of vinyl PDMS as a stabilizing agent in MMA dispersion polymerization reactions using $\mathrm{scCO}_{2}$.

FTIR analyses were conducted for the same PMMA samples, as shown in Fig. 4, also aiming to verify the efficiency of the process in forming PMMA, as well as to find some evidence of the nonreactive role of vinyl terminated PDMS in the system. Similarly to ${ }^{1} \mathrm{H}$ NMR analyses, one spectra is shown, since all other samples presented the same results. At the surfactant concentrations evaluated and for the system tested, there was no incorporation of the surfactant into the polymer chain, neither side reactions that could be detected by FTIR analysis, supporting the results observed by ${ }^{1} \mathrm{H}$ NMR.

Analyzing Fig. 4, it can be seen from the bands at $847 \mathrm{~cm}^{-1}$, $981 \mathrm{~cm}^{-1}$ and $1063 \mathrm{~cm}^{-1}$, characteristic absorption of PMMA, related to $\mathrm{C}-\mathrm{O}-\mathrm{C}$ deformation vibration. The band at $1443 \mathrm{~cm}^{-1}$ can be assigned to the $\mathrm{C}-\mathrm{H}$ deformation vibrations from the $\mathrm{CH}_{3}$ and $\mathrm{CH}_{2}$ groups. The band at $1730 \mathrm{~cm}^{-1}$ can be attributed to $\mathrm{C}=\mathrm{O}$ stretching vibration, from the acrylate carboxyl group. Absorption bands at $3003 \mathrm{~cm}^{-1}$ and $2941 \mathrm{~cm}^{-1}$ are related to $\mathrm{C}-\mathrm{H}$ stretching vibrations of the $\mathrm{CH}_{3}$ and $\mathrm{CH}_{2}$ groups. The bands at $1391 \mathrm{~cm}^{-1}$ and $754 \mathrm{~cm}^{-1}$ can be assigned to $\alpha$-methyl group vibrations. Finally, the absorption band in the region between 1150 and $1250 \mathrm{~cm}^{-1} \mathrm{can}$ be attributed to the $\mathrm{C}-\mathrm{O}-\mathrm{C}$ stretching vibration. FTIR results confirm, then, that the prepared polymer was indeed PMMA. Both results from ${ }^{1} \mathrm{H}$ NMR and FTIR are important to determine the adequacy of the process in forming PMMA, as well as of vinyl terminated PDMS to be used as a stabilizing agent for the polymerization of methyl methacrylate in supercritical $\mathrm{CO}_{2}$.

FTIR analysis was also performed for the vinyl PDMS surfactant, as shown in Fig. 5, in order to compare with the PMMA obtained and verify possible interaction that could have occurred. From the spectrum of vinyl PDMS it can be observed a band at $2960 \mathrm{~cm}^{-1}$ that can be attributed to $\mathrm{C}-\mathrm{H}$ stretching from $\mathrm{CH}_{3}$ groups. The weak absorbance at $1599 \mathrm{~cm}^{-1}$ can be assigned for $\mathrm{C}=\mathrm{C}$ double bond from vinyl group. The band at $1410 \mathrm{~cm}^{-1}$ can be assigned for the $=\mathrm{C}-\mathrm{H}$ bending vibration from vinyl groups and at $1257 \mathrm{~cm}^{-1}$ for the $\mathrm{CH}_{3}$ symmetric deformation of $\mathrm{Si}-\mathrm{CH}_{3}$ bond. Absorbances at 1021 and $1084 \mathrm{~cm}^{-1}$ can be attributed to $\mathrm{Si}-\mathrm{O}-\mathrm{Si}$ stretching vibrations. Finally, the band at $794 \mathrm{~cm}^{-1}$ is related to the $\mathrm{Si}-\mathrm{C}$ stretching. By analyzing both PMMA and vinyl PDMS spectra it is possible to suggest that no interaction between monomer and stabilizer, other than steric stabilization during polymerization reaction occurred, although further analyses are needed to confirm this finding.

\section{Conclusions}

In this work it was shown that it was possible to obtain poly (methyl methacrylate) through dispersion in supercritical $\mathrm{CO}_{2}$ using the surfactant vinyl terminated PDMS. The stabilizing agent was shown to be adequate for the stabilization of MMA polymerization system. Adjustments in stabilizer concentration might be appropriate in order to obtain monodispersed particles. PMMA molecular weight showed high values, in accordance to what is expected from dispersion in $\mathrm{scCO}_{2}$. Pressure fluctuations were observed in the first two hours of reactions, probably favored by the exothermic characteristic of organic free radical polymerization reactions. This may cause changes in the solubility of MMA in $\mathrm{CO}_{2}$, thus, influencing the polymer chain formation. The choice for this surfactant relies on the fact that it is a low cost stabilizer, in comparison to fluorinated compounds, commercially available and adequate for supercritical $\mathrm{CO}_{2}$ processing. Therefore, for scale up processes it might be a valuable alternative to fluorinated compounds and other siloxane based stabilizers. The reactional system proposed here was efficient. Adjustments in the procedure in order to eliminate pressure oscillations, as well as in the concentration of the reaction to optimize the system should be evaluated.

\section{Acknowledgements}

We gratefully acknowledge The University of Lorraine (ENSIC/ LRGP), The Federal University of Santa Catarina (EQA/LCP), the CNRS (Centre National de la Recherche Scientifique) for the technical assistance and support, and The Institut Carnot, France, for financial support. 


\section{REFERENCES}

[1] S.-H. Han, K.-K. Park, S.-H. Lee, GMA-functionalized reactive stabilizer for polymerization of methyl methacrylate in supercritical co2: effect of stabilizer, initiator and monomer concentrations, Macromol. Res. 16 (2) (2008) 120-127.

[2] S.-H. Han, K.-K. Par, S.-H. Lee, Polymerization of methyl methacrylate in carbon dioxide using glycidyl methacrylate linked reactive stabilizer: effect of pressure reaction time and mixing, Macromol. Res. 17 (2009) 51-57.

[3] E. Reverchon, R. Adami, S. Cardea, G. Della Porta, Supercritical fluids processing of polymers for pharmaceutical and medical applications: review, J. Supercrit. Fluids 47 (2009) 484-492.

[4] M.R. Giles, J.N. Hay, S.M. Howdle, R.J. Winder, Macromonomer surfactants for the polymerization of methylmethacrylate in supercritical $\mathrm{CO}_{2}$, Polymer 41 (2000) 6715-6721.

[5] A. Rosell, G. Storti, M. Morbidelli, dispersion polymerization of methyl methacrylate in supercritical carbon dioxide using a pseudo-graft stabilizer: role of reactor mixing, Macromolecules 37 (2004) 2996-3004.

[6] W. Wang, M.T. Griffiths, M.R. GileS, P. Williams, S.M. Howdle, Monitoring dispersion polymerizations of methyl methacrylate in supercritical carbon dioxide, Eur. Polym. J. 39 (2003) 423-428.
[7] J.M. De Simone, E.E. Maury, Y.Z. Menceloglu, J.B. McClain, T.J. Romack, J.R. Combes, Dispersion polymerizations in supercritical carbon dioxide, Science 265 (1994) 356-359.

[8] J.M. De Simone, H. Shiho, Dispersion polymerization of styrene in supercritical carbon dioxide utilizing random copolymers including fluorinated acrylate for preparing micron-size polystyrene particles, J. Polym. Sci. Part A 38 (2000) 1146-1153.

[9] S. Beuermann, M. Buback, Supercritical Carbon Dioxide in Polymer Reaction Engineering, Wiley-VCH, Weinheim, FRG, 2005, doi:http://dx.doi.org/10.1002/ 3527606726.fmatter.

[10] P. Lacroix-Desmazes, Polymérisations en milieu fluide supercritique, in: J.-C Daniel, C.H. Pichot (Eds.), Les Latex Synthétiques: élaboration et Applications, 33, Tec\&Doc-Lavoisier, Paris, 2006, pp. 911-933.

[11] J.-Y. Park, J.-J. Shim, Emulsion stability of PMMA particles formed by dispersion polymerization of methyl methacrylate in supercritical carbon dioxide, J. Supercrit. Fluids 27 (2003) 297-307.

[12] S. Diankov, D. Barth, A. Vega-Gonzalez, I. Pentchev, P. Subra-Paternault, Impregnation isotherms of hydroxybenzoic acid on PMMA in supercritical carbon dioxide, J. Supercrit. Fluids 41 (2007) 164-172.

[13] R. Wang, H.M. Cheung, A new PDMS macromonomer stabilizer for dispersion polymerization of styrene in supercritical carbon dioxide, J. Appl. Polymer Sci. 93 (2) (2004) 545-549. 